\title{
Universal behavior for magnetic entropy change in magnetocaloric materials: An analysis on the nature of phase transitions
}

\author{
Claudia Marcela Bonilla* \\ Instituto de Ciencia de Materiales de Aragón, Departamento de Física de la Materia Condensada, CSIC-Universidad de Zaragoza, \\ Pedro Cerbuna 12, 50009 Zaragoza, Spain \\ Julia Herrero-Albillos \\ Helmholtz-Zentrum Berlin für Materialien und Energie GmbH, Albert-Einstein-Str. 15, 12489 Berlin, Germany
}

Fernando Bartolomé, Luis Miguel García, and María Parra-Borderías

Instituto de Ciencia de Materiales de Aragón, Depto. de Física de la Materia Condensada, CSIC-Universidad de Zaragoza, Pedro Cerbuna 12, 50009 Zaragoza, Spain

Victorino Franco

Departamento de Física de la Materia Condensada, ICMSE-CSIC, Universidad de Sevilla, P.O. Box 1065, 41080 Sevilla, Spain

(Received 29 September 2009; revised manuscript received 21 April 2010; published 16 June 2010)

\begin{abstract}
A universal curve for the change in the magnetic entropy has been recently proposed for materials with second-order phase transitions. In this work we have studied the universal behavior of the magnetocaloric effect in the family of cobalt Laves phases, $R \mathrm{Co}_{2}$, and mixed manganites, $\mathrm{La}_{2 / 3}\left(\mathrm{Ca}_{x} \mathrm{Sr}_{(1-x)}\right)_{1 / 3} \mathrm{MnO}_{3}$, which exhibit first- and second-order phase transitions. The rescaled magnetic entropy change curves for different applied fields collapse onto a single curve for materials with second-order phase transition as opposed to the first-order phase transition compounds, for which this collapse does not hold. This result suggests that the universal curve may be used as a further criterion to distinguish the order of the phase transition.
\end{abstract}

DOI: 10.1103/PhysRevB.81.224424

PACS number(s): 75.30.Sg, 77.80.B-, 71.20.Eh

\section{INTRODUCTION}

Magnetic refrigerators are a new environmentally friendly option to the conventional devices based on compression expansion of gases. These devices also show higher energy efficiency than those based on ozone-depleting gases. The physical basis behind the operation of this kind of equipment is the magnetocaloric effect (MCE). Currently, the development of this technology is tied to the research in materials presenting optimal magnetocaloric properties (namely, large magnetic entropy change, $\Delta S_{M}$, and large refrigerant capacity, $\mathrm{RC}$ ) near room temperature.

The RC of a given refrigerant is defined as the area below the $\Delta S_{M}(T)$ curve between the temperatures of the cold and hot reservoirs ( $T_{\text {cold }}$ and $T_{\text {hot }}$, respectively). ${ }^{1} T_{\text {cold }}$ and $T_{\text {hot }}$ are usually taken as those temperatures where $\Delta S_{M}$ equals $\Delta S_{M}^{\text {peak }} / 2$. Therefore, in order to get high RC values both the height and the width of the $\Delta S_{M}$ peak have to be considered.

Materials presenting first-order magnetostructural phase transitions frequently show giant magnetocaloric effect (GMCE), ${ }^{2}$ i.e., very large values of $\Delta S^{\text {peak }}$. However, firstorder phase transitions have two important drawbacks, namely, the narrowness of the $\Delta S_{M}$ curve and the presence of hysteresis, which leads to low operation frequencies and cooling power. ${ }^{3}$ To overcome these problems, compounds undergoing second-order phase transitions may be used. Although these compounds do present smaller $\left|\Delta S_{M}^{\text {peak }}\right|$ than GMCE materials, they do not show thermal hysteresis and their $\Delta S_{M}(T)$ is extended through a wider temperature range. The compromise between an optimal RC and the lack of hysteresis makes compounds with second-order phase transitions better candidates for the development of magnetic cooling devices at the present moment.
The MCE is frequently characterized by measuring magnetization $M(H)$ curves at different temperatures, allowing $\Delta S_{M}(T)$ to be obtained by means of the Maxwell relations. Recently, V. Franco et al. ${ }^{4-6}$ have described the universal behavior for the $\Delta S_{M}(T)$ in compounds with second-order phase transition. In addition to the intrinsic beauty of a universal behavior, this curve allows the prediction of the field dependence of $\Delta S_{M}(T)$ even in those materials that do not follow a mean-field approach; and it can be used to make extrapolations in temperature or field close to the entropy change peak. From a theoretical point of view, the universal curve can be derived from the equation of state and the critical exponents of the system; from a practical point of view, the phenomenological approach allows to construct the universal curve without knowing the critical exponents or the equation of state for the material under study.

The universal behavior of $\Delta S_{M}$ has been confirmed in several second-order transition compounds, including $\mathrm{Fe}$ based amorphous alloys such as $\mathrm{FeMoCuB},{ }^{7} \mathrm{FeCrMoBCu},{ }^{8,9}$ and $\mathrm{FeZrBCu},{ }^{10}$ in $\mathrm{Gd}$ and the intermetallic $\mathrm{Er}_{1-x} \mathrm{Dy}_{x} \mathrm{Al}_{2},{ }^{11}$ and in $\mathrm{TbCo}_{2},{ }^{12}$ among others.

It is interesting to note that the collapse of these curves is observed not only in the near vicinity of the transition but in a wide temperature range. This raises the question as to whether the collapse of the $\Delta S_{M}(T)$ curves is a manifestation of a universal behavior or not. A study on first-order phase transitions should shed light on the subject: a breakdown of the universal curve is expected for first-order phase transitions if the underlying cause is universality associated to critical phenomena and intrinsic to second-order phase transitions. Otherwise, $\Delta S(T)$ curves may collapse in the same way for first order as for second-order transitions. 
The aim of this work is to systematically study the behavior of this universal curve for the magnetic entropy change for two families of compounds, which present both first- and second-order phase transitions. Indeed, we aim at showing whether or not a breakdown of the universal behavior of $\Delta S_{M}$ occurs in first-order phase transitions.

We have chosen the cobalt Laves phases family due to its rich phenomenology: first, compounds formed with light rare-earth ions are ferromagnets while those formed with heavy rare earths are ferrimagnets. Second, the magnetic order is established through a second-order phase transition in all of them except in $\mathrm{ErCo}_{2}, \mathrm{HoCo}_{2}$, and $\mathrm{DyCo}_{2}$, where the magnetic ordering is coupled to a structural change, leading to a first-order magnetostructural transition. Additionally the ferrimagnetic $\mathrm{HoCO}_{2}$ and ferromagnetic $\mathrm{NdCo}_{2}$ undergo a first-order spin reorientation transition (SRT) below their magnetic ordering transitions. The structural, electronic, and magnetic properties of this family of compounds have been thoroughly studied. ${ }^{13,14}$ Moreover, the large entropy change showed by $\mathrm{ErCo}_{2}$ has led to studies on the MCE properties of the pure and pseudobinary Co Laves phases. ${ }^{2,15-22}$

We have also selected the ferromagnetic manganites, $\mathrm{La}_{2 / 3}\left(\mathrm{Ca}_{x} \mathrm{Sr}_{(1-x)}\right)_{1 / 3} \mathrm{MnO}_{3}$ with $x=0,0.5$, and 1 , in order to give more generality to our results. The physical properties of these materials have been reported in literature. ${ }^{23-27}$ A magnetic transition with first-order character has been determined for $\mathrm{La}_{2 / 3} \mathrm{Ca}_{1 / 3} \mathrm{MnO}_{3}$ at $260 \mathrm{~K}$ while $\mathrm{La}_{2 / 3}\left(\mathrm{Ca}_{0.5} \mathrm{Sr}_{0.5}\right)_{1 / 3} \mathrm{MnO}_{3}$ and $\mathrm{La}_{2 / 3} \mathrm{Sr}_{1 / 3} \mathrm{MnO}_{3}$ show a secondorder transition at $340 \mathrm{~K}$ and $370 \mathrm{~K}$, respectively. ${ }^{23,24}$

\section{EXPERIMENTAL DETAILS AND DATA ANALYSIS}

Intermetallic samples of $R \mathrm{Co}_{2}$ with $R=\mathrm{Tb}, \mathrm{Pr}, \mathrm{Nd}$, Dy, and Ho were prepared by melting the pure metallic precursors in an induction furnace under Ar atmosphere. The alloys were later annealed under $\mathrm{Ar}$ atmosphere at $850{ }^{\circ} \mathrm{C}$ for $8-12$ days depending on the sample. The policrystalline manganites $\mathrm{La}_{2 / 3}(\mathrm{Ca}, \mathrm{Sr})_{1 / 3} \mathrm{MnO}_{3}$ were obtained from $\mathrm{La}_{2} \mathrm{O}_{3}, \mathrm{CaCo}_{3}$, $\mathrm{Mn}_{2} \mathrm{O}_{3}$, and $\mathrm{Sr}_{2} \mathrm{Co}_{3}$ as precursors. The starting powders were ground, pelleted, and sintered following a standard ceramic method. ${ }^{24}$ A highly pure single phase was found in all the samples as checked by x-ray diffraction.

Field dependence of magnetization measurements were performed in a Quantum Design MPMS-5S superconducting quantum interference device magnetometer. $M(H)$ isotherms were obtained by varying the field between 0 to $5 \mathrm{~T}$ for all samples. Between 30 and $70 M(H)$ curves were measured in a range from 6 to $400 \mathrm{~K}$, depending on the sample ordering temperature, $T_{c}$, and the presence of SRT.

The magnetic ordering at zero field occurs at $40 \mathrm{~K}, 78 \mathrm{~K}$, $98 \mathrm{~K}, 138 \mathrm{~K}$, and $231 \mathrm{~K}$ for $\mathrm{PrCo}_{2}, \mathrm{HoCo}_{2}, \mathrm{NdCo}_{2}, \mathrm{DyCo}_{2}$, and $\mathrm{TbCo}_{2}$, respectively. The SRT temperature $T_{\mathrm{SRT}}$ for $\mathrm{NdCo}_{2}$ and $\mathrm{HoCo}_{2}$ are $42 \mathrm{~K}$ and $16 \mathrm{~K}$, respectively. The magnetic ordering temperatures for $\mathrm{La}_{2 / 3} \mathrm{Ca}_{1 / 3} \mathrm{MnO}_{3}$, $\mathrm{La}_{2 / 3} \mathrm{Sr}_{1 / 3} \mathrm{MnO}_{3}$, and $\mathrm{La}_{2 / 3}\left(\mathrm{Ca}_{0.5} \mathrm{Sr}_{0.5}\right)_{1 / 3} \mathrm{MnO}_{3}$ were identified as $260 \mathrm{~K}, 340 \mathrm{~K}$, and $370 \mathrm{~K}$, respectively. These data are fully consistent with those previously reported. ${ }^{23,24,28}$

The magnetic entropy change $\Delta S_{M}(T)$ can be obtained from the $M(H)$ curves by applying a numerical approximation to the equation
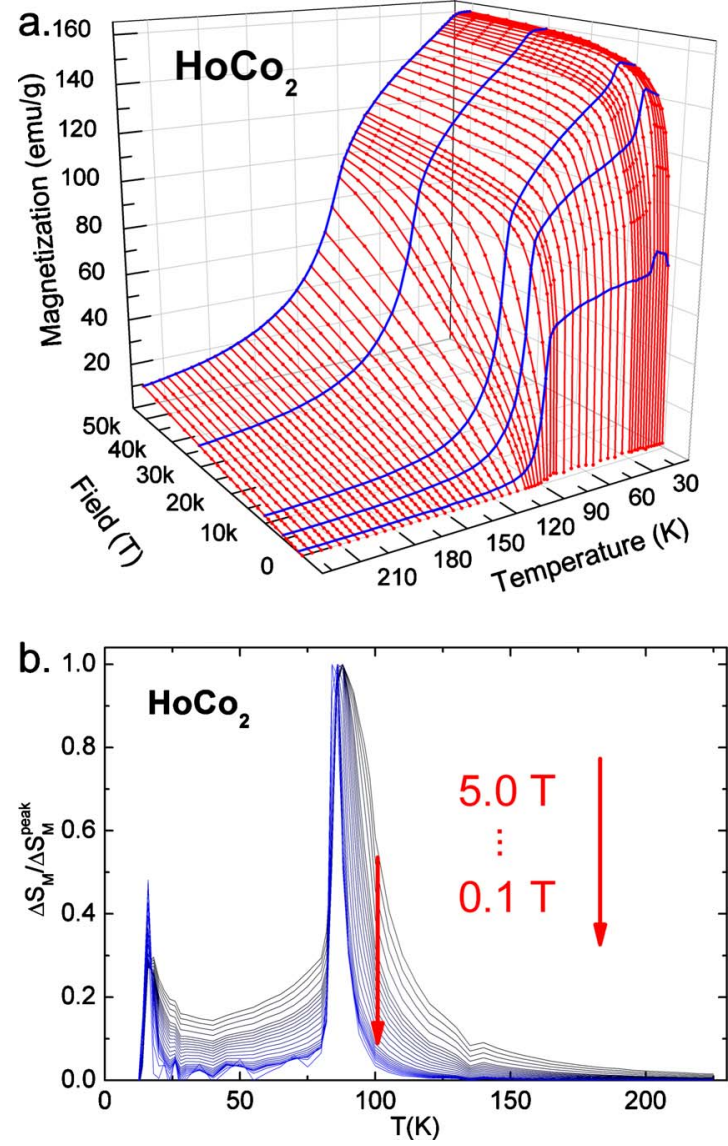

FIG. 1. (Color online) (a) Magnetization measurements as function of field for different temperatures for $\mathrm{HoCO}_{2}$. The values of applied field during the measurement were $0,0.1,0.2,0.3,0.4,0.5$, $0.6,0.7,0.9,1.0,1.2,1.4,1.6,1.8,2.0,2.2,2.4,2.6,2.8,3.0,3.5$, $4.0,4.5$, and $5.0 \mathrm{~T}$. (b) Normalized entropy change versus temperature for different applied fields for $\mathrm{HoCo}_{2}$.

$$
\Delta S_{M}=\int_{0}^{H}\left(\frac{\partial M}{\partial T}\right)_{H} d H
$$

replacing the partial derivative for finite differences and numerically solving the integrals for each value of $H$.

In Fig. 1 panel a we can observe the $M(H)$ curves measured for $\mathrm{HoCo}_{2}$ for 58 values of temperature between 6 and $225 \mathrm{~K}$. Correspondingly panel b shows the $\Delta S_{M}(T)$ curves normalized to their maximum value $\Delta S^{\text {peak }}$ for 25 field values.

The construction of the phenomenological universal curve is based on the collapse of the $\Delta S_{M}(T, H)$ points corresponding to equivalent states of the system into one single point in the new curve. Those equivalent states have the same height, $h$, in the $\Delta S_{M} / \Delta S_{M}^{\text {peak }}$ curves. For each value of the applied field and any arbitrary value of $h$, two reference temperatures $\left(T_{r 1}<T_{c}\right.$ and $\left.T_{r 2}>T_{c}\right)$ are found so that $\Delta S_{M}\left(T_{r 1}\right) / \Delta S_{M}^{p e a k}$ $=\Delta S_{M}\left(T_{r 2}\right) / \Delta S_{M}^{p e a k}=h$. The collapse of the normalized entropy change curves can be then obtained by defining a new variable for the temperature axis, $\theta$, given by the expression 


$$
\theta=\left\{\begin{array}{cc}
-\left(T-T_{c}\right) /\left(T_{r_{1}}-T_{c}\right) & T \leq T_{c} \\
\left(T-T_{c}\right) /\left(T_{r_{2}}-T_{c}\right) & T>T_{c} .
\end{array}\right.
$$

In this work we have identified $T_{c}$ as the temperature of the maximum entropy change ${ }^{29}$ and we have selected $h=0.5$ when constructing the universal curve for each sample. By construction, the temperature axis is rescaled in a different way below and above $T_{c}$ imposing the constraint that the reference points in the new curve correspond to $\Delta S_{M}(\theta$ $= \pm 1) / \Delta S_{M}^{\text {peak }}=h$.

The existence of the universal curve for second-order phase transitions has been already theoretically grounded. ${ }^{30}$ The assumption that different physical magnitudes (such as magnetization) scale, in the vicinity of a second-order transition, is well supported both theoretically and experimentally. ${ }^{31}$ Based on this statement we can consider the scaling equation for a magnetic system given by ${ }^{32}$

$$
\frac{H}{M^{\delta}}=h\left(\frac{t}{M^{1 / \beta}}\right),
$$

where $M$ is the magnetization, $H$ is the applied field, $t$ is the reduced temperature, $\delta$ and $\beta$ are critical exponents for the critical isotherm $(t=0)$ and the magnetization behavior along coexistence $(H=0, t<0)$, respectively, and $h(x)$ is a scaling function. This $h(x)$ is the same for systems belonging to the same universality class provided that the magnetization and magnetic field units are such that $h(0)=1$ and $h(-1)=0$. The Eq. (3) can be written as

$$
\frac{M}{|t|^{\beta}}=m_{ \pm}\left(\frac{H}{|t|^{\Delta}}\right)
$$

the product $\beta \delta=\Delta$, determines the gap exponent and the \pm sign is related to $t>0$ and $t<0$, respectively.

Combining Eqs. (2) and (4) and after some algebra ${ }^{30}$ the entropy change can be expressed as

$$
\begin{aligned}
\Delta S_{M} / a_{M} & = \pm|t|^{1-\alpha} \int_{0}^{H / t||^{\Delta}} d x\left[\beta m_{ \pm}(x)-\Delta x m_{ \pm}^{\prime}(x)\right] \\
& =|t|^{1-\alpha} \widetilde{S}\left(t / H^{1 / \Delta}\right)=H^{1-\alpha / \Delta} s\left(t / H^{1 / \Delta}\right),
\end{aligned}
$$

where $a_{M}=T_{c}^{-1} A^{\delta+1} B$, with $A$ and $B$ the critical amplitudes at coexistence $\left[M=A(-t)^{\beta}\right]$ and along the critical isotherm $(H$ $\left.=B M^{\delta}\right)$, respectively. Here $s(x)$ is the scaling function. If the reduced temperature $t$ is rescaled by a factor proportional to $H^{1 / \Delta}$, and the magnetic entropy change by $a_{M} H^{(1-\alpha) / \Delta}$ the expression (5) shows that the experimental data would collapse onto the same curve. This demonstration proves that the MCE data of different alloys belonging to the same universality class should collapse in a common universal curve.

In this way the universal curve can also be constructed analytically if the equation of state and the critical exponents of a material are known. However, from a practical point of view, the phenomenological approach allows the use of the universal curve for practical purposes without knowing those details about the material.

A single reference temperature can be used to collapse all the curves. ${ }^{30}$ However, the use of two reference temperatures has been necessary in some special cases to obtain a satis- factory universal curve. In particular to correct the presence of a minority magnetic phase in $\mathrm{LaFe}_{10.8} \mathrm{Si}_{2.2}$ (Ref. 12) or the influence of the demagnetization factor. ${ }^{33}$ In the present work, we have used two references instead of one in order to assure that if a breakdown of the universal behavior of $\Delta S_{M}$ should occur, it could not be ascribed to any of the previously mentioned artifactual causes.

\section{RESULTS AND DISCUSSION}

The normalized entropy change as a function of the rescaled temperature $\theta$ for the magnetic ordering transitions of the $R \mathrm{Co}_{2}$ and the mixed manganites compounds are shown in Figs. 2 and 3, respectively.

Panels a and b of Fig. 2 show our results for the secondorder phase transitions of $\mathrm{TbCo}_{2}$ and $\mathrm{PrCo}_{2}$. The results for the second-order transitions in the mixed manganites family, $\mathrm{La}_{2 / 3} \mathrm{Sr}_{1 / 3} \mathrm{MnO}_{3}$ and $\mathrm{La}_{2 / 3}\left(\mathrm{Ca}_{0.5} \mathrm{Sr}_{0.5}\right)_{1 / 3} \mathrm{MnO}_{3}$, are presented, respectively, in panel $a$ and $b$ of Fig. 3. The collapse of all these data into a unique curve-in a very wide temperature range-for the $\mathrm{RCO}_{2}$ compounds and the manganites is a further confirmation of the general validity of the treatment in second-order phase transition compounds. Indeed, the universal behavior of $\Delta S_{M}$ had been independently demonstrated for another $\mathrm{TbCo}_{2}$ sample. ${ }^{12}$

Panel d of Fig. 2 shows the result for $\mathrm{DyCo}_{2}$. From mere inspection of the graph, it is evident that-for temperatures below $T_{c}$-the curves do not overlap, pointing out that this alloy does not follow a universal curve for magnetic entropy change. In the case of ferromagnetic first-order phase transition of $\mathrm{La}_{2 / 3} \mathrm{Ca}_{1 / 3} \mathrm{MnO}_{3}$ (see panel c in Fig. 3) a breakdown of the universal behavior for the normalized entropy change can be observed.

The collapse for $\theta>0$ is due to the paramagnetic behavior. Magnetization scales with $(\mu H / k T)$ and therefore it is possible to collapse $\Delta S$ for every compound in the paramagnetic region. For values $-1<\theta<0$ the deviation from collapse cannot be very large, as the curves coincide by construction. The reference points are such that $\Delta S_{M}(\theta$ $= \pm 1) / \Delta S_{M}^{\text {peak }}=h$, where $h$ is arbitrary $(0<h<1)$, in consequence the collapse is broken only below $\theta=-1$. Within the range $-1<\theta<0$ the collapse is real in second-order transitions and only apparent in first-order transitions. Therefore, the effect of the order of the transition is decisive only below $\theta=-1$, in this phenomenological approach. In principle, the presence of a minority magnetic phase in the sample, or the demagnetizing factor could be responsible of an apparent breakdown of the universal curve. ${ }^{12,33}$ However, as was pointed out previously, two reference temperatures have been used throughout this work and therefore the effect of those phenomena have been excluded. For this reason, we ascribe the breakdown of the universal curve in $\mathrm{DyCo}_{2}$ and $\mathrm{La}_{2 / 3} \mathrm{Ca}_{1 / 3} \mathrm{MnO}_{3}$ to the first-order nature of their phase transitions.

Furthermore, the observed behavior in first-order phase transitions suggests that the collapse of the $\Delta S_{M}$ curves is related to the universality intrinsic to second order phase transitions. ${ }^{31}$ The breakdown of the universal behavior can be quantified from the vertical spread of the points for values 

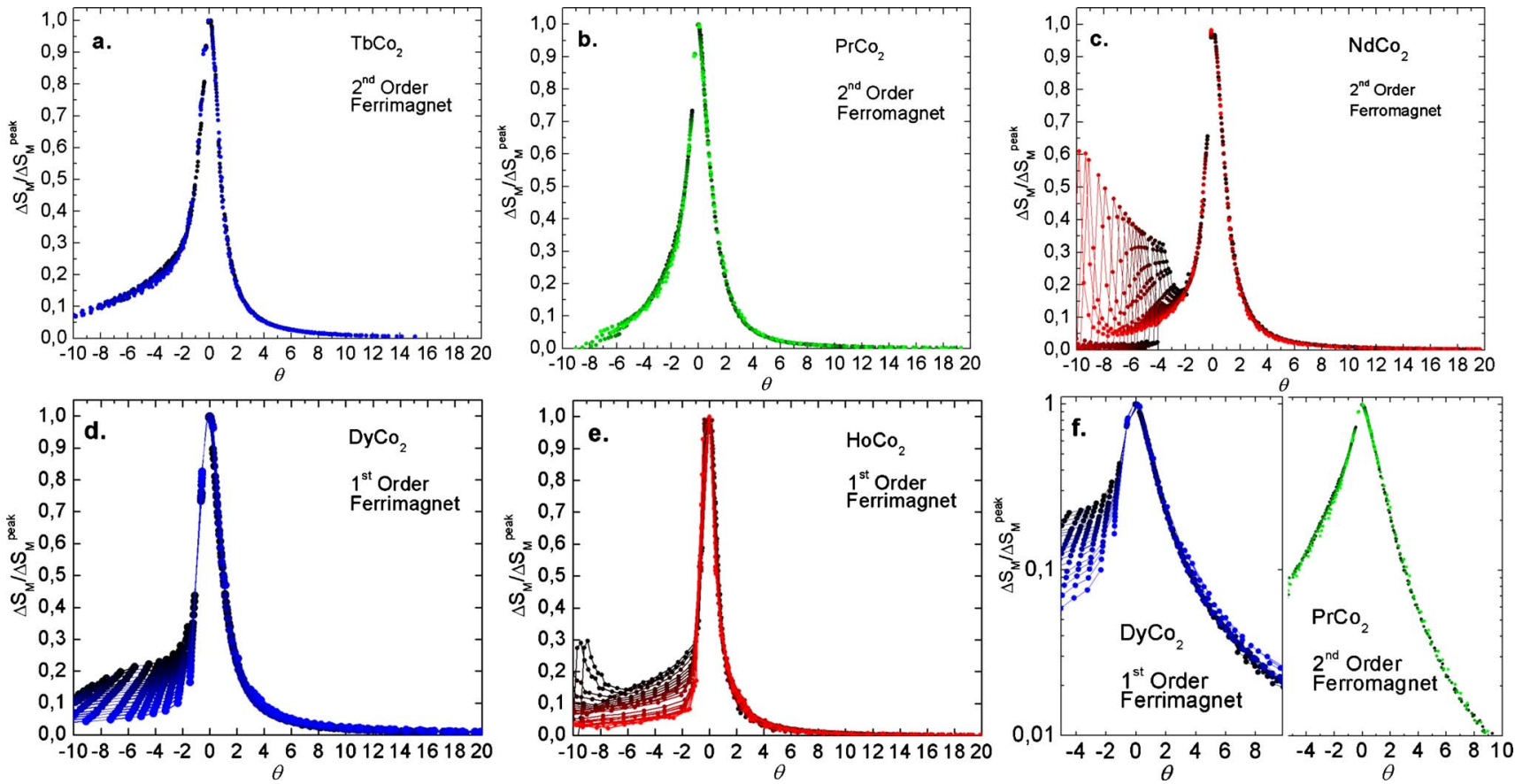

FIG. 2. (Color online) Normalized entropy change as a function of the rescaled temperature $\theta$ for the cobalt Laves phases studied in this work. A universal curve for the second-order phase transitions of $\mathrm{TbCo}_{2}$ (panel a), $\mathrm{PrCo}_{2}$ (panel b), and $\mathrm{NdCo}_{2}$ (panel c) is demonstrated while a breakdown of the universal curve for the first order phase transitions of $\mathrm{DyCo}_{2}$ (panel d) and $\mathrm{HoCo}_{2}$ (panel e) can be observed. The panel $\mathrm{f}$ shows a comparison of the rescaled curves for $\mathrm{PrCo}_{2}$ and $\mathrm{DyCo}_{2}$ (vertical axis in logarithmic scale).

below $\theta=-1$. We have calculated the width $W$ of the vertical spreading of each scaled entropy change curve relative to its mean value at an arbitrarily chosen $\theta<-1$. The dispersion is then given by

$$
\text { dispersion }=100 \times \frac{W(\theta=-5)}{\Delta S_{M} / \Delta S_{M}^{\text {peak }}(\theta=-5)} .
$$

In Table I we list the values obtained for the dispersion in the studied compounds. Clearly, for compounds with first-order phase transition the dispersion always remain superior to $100 \%$, i.e., the width of the vertical spreading is larger than the mean value of $\Delta S_{M} / \Delta S_{M}^{\text {peak }}$ for that value of $\theta$. For compounds with second-order phase transition the dispersion is never larger than $30 \%$, which may be due to the experimental uncertainty. From our results, we expect this behavior holds regardless the family of compounds.

The compounds $\mathrm{NdCo}_{2}$ and $\mathrm{HoCo}_{2}$ are selected to highlight the influence of the thermodynamical order of the transition on whether or not the rescaled entropy curves collapse into a universal behavior. $\mathrm{NdCO}_{2}$ shows a typical ferromagnetic second-order phase transition at $T_{c}=95 \mathrm{~K}$ and a SRT of first order at lower temperature $\left[T_{\mathrm{SRT}} \sim 42 \mathrm{~K}\right.$ (Ref. 20)]. As is shown in panel c of Fig. 2-where $\theta=0$ corresponds to $T_{c}$ - the expected collapse for a second-order phase transition is observed except in the vicinity of the SRT. The observed shift of the SRT peaks is due to: first, the usual dependence of the critical temperature on the applied field in first-order phase transitions $\mathrm{s}^{20}$ and second — and more significantly—due to the scaling around $T_{c}$.
The situation is similar for $\mathrm{HoCo}_{2}$ where a ferrimagnetic transition occurs at $T_{c}=86 \mathrm{~K}$ and the SRT takes place at $T_{\mathrm{SRT}} \sim 16 \mathrm{~K}$ (Ref. 20) although for this system both are firstorder phase transitions. Panel e of Fig. 2-where again $\theta$ $=0$ corresponds to $T_{c}$ - shows the breakdown of the universal curve for the $T_{c}$ of $\mathrm{HoCo}_{2}$. Below $T_{c}$, the rescaled $\Delta S_{M}$ curves show a behavior very similar to that observed in $\mathrm{DyCo}_{2}$. Further splitting of the $\Delta S_{M}(\theta)$ curves at lower temperatures $(\theta<-6)$ comes from the SRT contribution, as in $\mathrm{NdCo}_{2}$.

It is now interesting to compare the result of scaling the $\Delta S_{M}$ curves for the different compounds. On one hand, as it is shown in panels a-c of Fig. 4, there is a common collapse within the scaled entropy change curves for second-order phase transition compounds. For $\mathrm{TbCo}_{2}, \mathrm{PrCo}_{2}$, and $\mathrm{NdCo}_{2}$, showed in panel a, the collapse to a common curve is satisfied except at lower $\theta$. The existence of a universality for the $\Delta S_{M}$ curves relies on the scaling with temperature of the magnetization (and, consequently, of the magnetic entropy) near a second-order phase transition. ${ }^{30,31}$ Therefore, every system from the same universality class, i.e., with the same critical exponents, will collapse into a common curve. Due to the fact that $\mathrm{TbCo}_{2}$ is a ferrimagnet while $\mathrm{PrCo}_{2}$ and $\mathrm{NdCo}_{2}$ are ferromagnets the common collapse is not satisfied. On the other side panels b and c of Fig. 4 show the comparison between ferromagnets: within the manganite family and for $\mathrm{La}_{2 / 3}\left(\mathrm{Ca}_{0.5} \mathrm{Sr}_{0.5}\right)_{1 / 3} \mathrm{MnO}_{3}$ and $\mathrm{PrCo}_{2}$ systems, respectively. The result is fully consistent with our previous statement. Second-order paramagnetic-ferromagnetic phase transition of double exchange materials close to half-filling [which is the case of manganites $\mathrm{La}_{2 / 3}\left(\mathrm{Ca}_{x} \mathrm{Sr}_{1-x}\right)_{1 / 3} \mathrm{MnO}_{3}$ ] belongs to a Heisenberg three-dimensional universality class $^{34,35}$ as well 

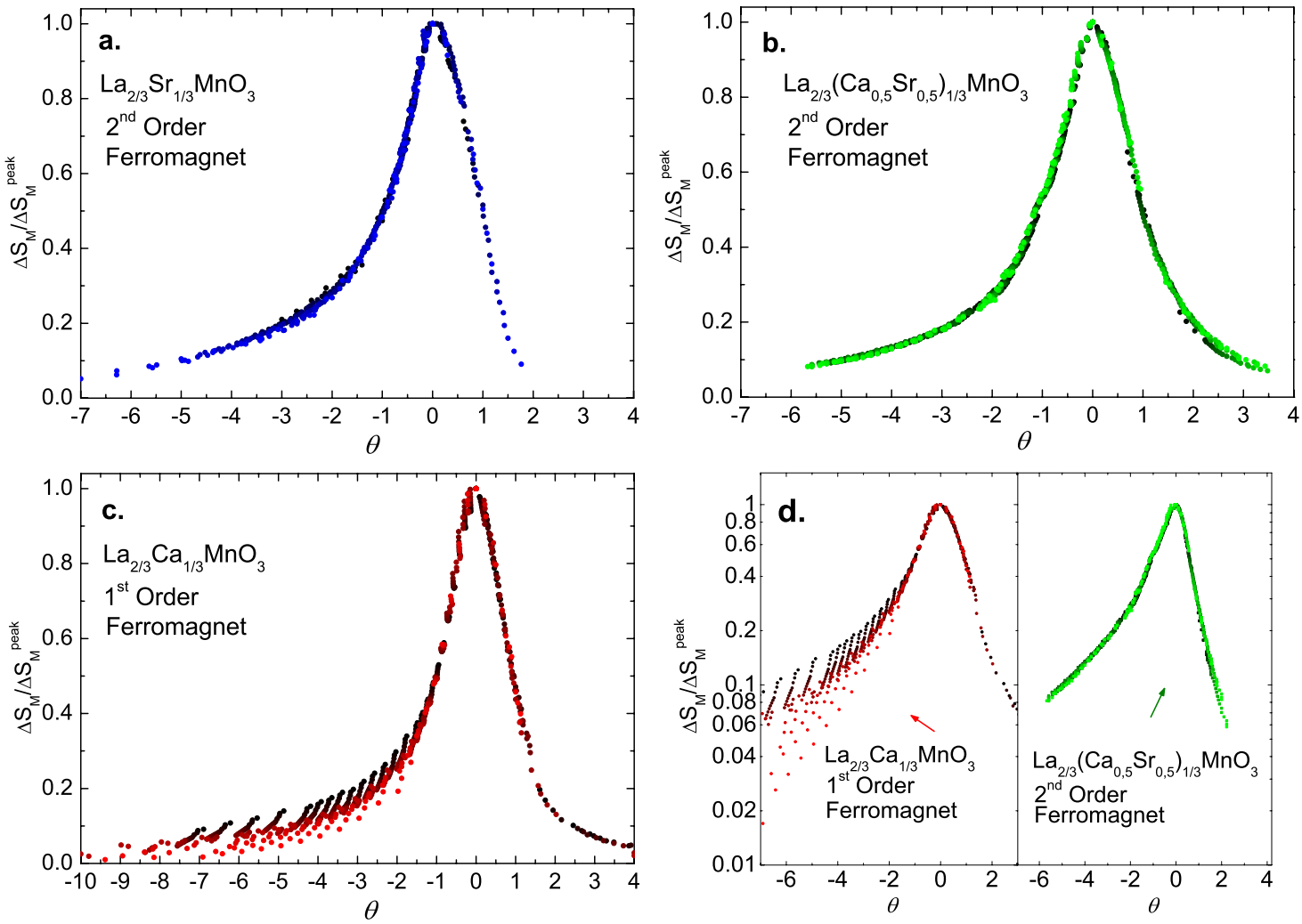

FIG. 3. (Color online) Normalized entropy change as a function of the rescaled temperature $\theta$ for the manganites studied in this work. A universal curve for the second-order phase transitions of $\mathrm{La}_{2 / 3} \mathrm{Sr}_{1 / 3} \mathrm{MnO}_{3}$ (panel a) and $\mathrm{La}_{2 / 3}\left(\mathrm{Ca}_{0.5} \mathrm{Sr}_{0.5}\right)_{1 / 3} \mathrm{MnO}_{3}$ (panel b), is demonstrated, while a breakdown of the universal curve for the first order phase transitions of $\mathrm{La}_{2 / 3} \mathrm{Ca}_{1 / 3} \mathrm{MnO}_{3}($ panel c). Panel d shows a comparison of the rescaled curves for $\mathrm{La}_{2 / 3} \mathrm{Ca}_{1 / 3} \mathrm{MnO}_{3}$ and $\mathrm{La}_{2 / 3}\left(\mathrm{Ca}_{0.5} \mathrm{Sr}_{0.5}\right)_{1 / 3} \mathrm{MnO}_{3}$ (vertical axis in logarithmic scale).

as second-order ferromagnets $\mathrm{RCo}_{2} \cdot{ }^{36} \mathrm{In}$ consequence the scaled entropy change curves for $\mathrm{La}_{2 / 3} \mathrm{Sr}_{1 / 3} \mathrm{MnO}_{3}$ and $\mathrm{La}_{2 / 3}\left(\mathrm{Ca}_{0.5} \mathrm{Sr}_{0.5}\right)_{1 / 3} \mathrm{MnO}_{3}$ do collapse in good approximation to a common behavior; as well as the curves for $\mathrm{PrCo}_{2}$ and $\mathrm{La}_{2 / 3}\left(\mathrm{Ca}_{0.5} \mathrm{Sr}_{0.5}\right)_{1 / 3} \mathrm{MnO}_{3}$ regardless both systems belong to different families of compounds.

Panel $\mathrm{f}$ of Fig. 2 and panel d of Fig. 3 shows the scaled entropy changes in semilogarithmic axis for $\mathrm{PrCo}_{2}$ and $\mathrm{DyCo}_{2}$ and $\mathrm{La}_{2 / 3} \mathrm{Ca}_{1 / 3} \mathrm{MnO}_{3}$ and $\mathrm{La}_{2 / 3}\left(\mathrm{Sr}_{0.5} \mathrm{Ca}_{0.5}\right)_{1 / 3} \mathrm{MnO}_{3}$, respectively. Both figures allow direct comparison between the results for a first- and a second-order phase transition in each family.

The abruptness of the changes in physical magnitudes at the first-order transition of $\mathrm{DyCo}_{2}$ are small, making its as-

TABLE I. Dispersion for scaled entropy change values at $\theta=$ -5 .

\begin{tabular}{lcc}
\hline \hline Order & Compound & Dispersion $(\%)$ \\
\hline First order & $\mathrm{DyCo}_{2}$ & 116.41 \\
& $\mathrm{HoCo}_{2}$ & 131.31 \\
& $\mathrm{La}_{2 / 3} \mathrm{Ca}_{1 / 3} \mathrm{MnO}_{3}$ & 105.55 \\
Second order & $\mathrm{TbCo}_{2}$ & 17.17 \\
& $\operatorname{PrCo}_{2}$ & 26.74 \\
& $\mathrm{La}_{2 / 3}\left(\mathrm{Sr}_{0.5} \mathrm{Ca}_{0.5}\right)_{1 / 3} \mathrm{MnO}_{3}$ & 9.09 \\
& $\mathrm{La}_{2 / 3} \mathrm{Sr}_{1 / 3} \mathrm{MnO}_{3}$ & 14.00 \\
\hline
\end{tabular}

cription as first order very difficult by inspection of experimental results alone. Usually the order of the transition can be distinguished from different experimental techniques such as specific heat, differential scanning calorimetry or resistivity, among others. However, these experiments usually involve long and careful measurements very near $T_{c}$. Moreover, conventional calorimetric measurements for samples of nanoscopic sizes are not sensitive enough to follow the rapid changes in temperature for such sample sizes. ${ }^{37}$ Even for bulk $\mathrm{DyCo}_{2}$ samples the establishment on the order of its transition is not straightforward. First, the jump in resistivity at the transition is small and not very abrupt, ${ }^{2}$ and the temperature range of metastability is very narrow. ${ }^{22}$ Additionally, an applied pressure well below 1GPa (Refs. 38 and 39) or a chemical dilution of $20 \%$ with $\mathrm{Tb}$, for example, ${ }^{40}$ are enough to destabilize the first-order character of the transition. All these results suggest that $\mathrm{DyCo}_{2}$ is a first order case near the critical point, i.e., on the border of second-order phase transition.

A criterion from purely magnetic measurements can be proposed as an alternative to calorimetric techniques. Usually the Banerjee criterion ${ }^{41}$ has been employed to establish the magnetic phase transition character. By studying the presence of a negative slope region on the isothermal plots of $H / M$ versus $M^{2}$ first-order phase transitions can be identified. In Fig. 5 we show that the criterion is clear for all the $R \mathrm{Co}_{2}$ but $\mathrm{DyCo}_{2}$. Indeed, neither magnetization nor Arrot plots allow clear determination of the order of that phase 

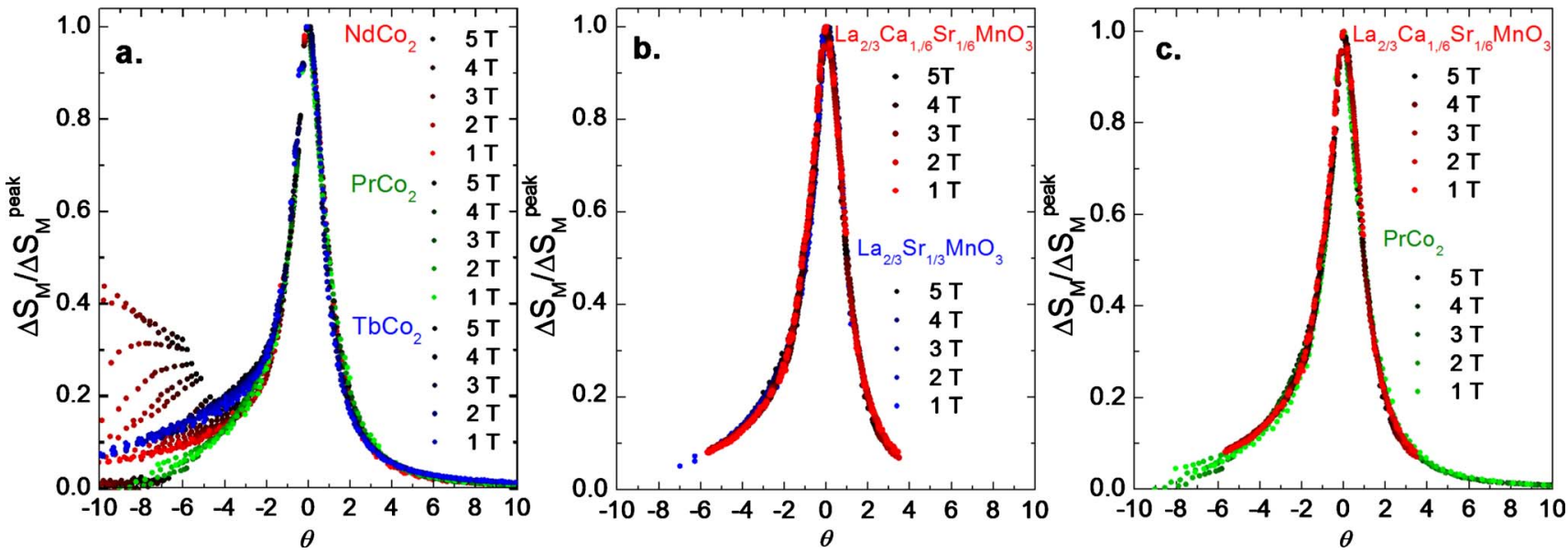

FIG. 4. (Color online) Universal behavior of the entropy change for the second-order phase transition compounds. (In panel a) Results for Co Laves phases. (In panel b) Results for mixed manganites family. (In panel c) Comparison between second order phase transition systems from different families of compounds, $\mathrm{PrCo}_{2}$ and $\mathrm{La}_{2 / 3}\left(\mathrm{Ca}_{0.5} \mathrm{Sr}_{0.5}\right)_{1 / 3} \mathrm{MnO}_{3}$.

transition in $\mathrm{DyCO}_{2} \cdot{ }^{20,22,40}$ The left panel of Fig. 5 shows that the slope of $H / M$ versus $M^{2}$ curve for $\mathrm{DyCo}_{2}$ is positive, contrary to what is expected for a first-order phase transition. This result is emphasized in the right panel of Fig. 5, where plots of the derivative of $H / M$ versus $M^{2}$ are presented. This result shows the difficulty in determining the order of the transition from purely magnetic measurements in compounds with small size of the discontinuities of the free energy derivatives at the transition. However, in panel $\mathrm{f}$ of Fig. 2, the breakdown of the universal behavior below $\theta$ $=0$ for $\mathrm{DyCo}_{2}$ is evident by mere inspection. Mixed manganites $\mathrm{La}_{2 / 3} \mathrm{Ca}_{1 / 3} \mathrm{MnO}_{3}$ and $\mathrm{La}_{2 / 3}\left(\mathrm{Ca}_{0.5} \mathrm{Sr}_{0.5}\right)_{1 / 3} \mathrm{MnO}_{3}$ show the same clear difference between the behaviors for first- and second-order transitions, (see panel d of Fig. 3).

These results allows us to propose the construction of the scaled $\Delta S_{M}$ curves as a way to discriminate the order of magnetic phase transitions by magnetic measurements only, as an alternative to the Banerjee criterion. ${ }^{22,24,41}$ Banerjee criterion is developed in the frame of Landau theory and it holds within a mean-field model. ${ }^{41}$ However, our method is more general in the sense that the scaling of the entropy change curves derives from the scaling of the order parameter, inherent to second-order phase transitions. ${ }^{31}$

\section{CONCLUSIONS}

A universal curve for the entropy change for compounds with second-order phase transition, within the Co Laves Phases- $\mathrm{TbCo}_{2}, \quad \mathrm{PrCo}_{2}$, and $\mathrm{NdCO}_{2}$ - and mixed manganites- $\mathrm{La}_{2 / 3} \mathrm{Sr}_{1 / 3} \mathrm{MnO}_{3}$ and $\mathrm{La}_{2 / 3}\left(\mathrm{Ca}_{0.5} \mathrm{Sr}_{0.5}\right)_{1 / 3} \mathrm{MnO}_{3}$ - can be constructed. Indeed a unique universal behavior is found for compounds with second-order phase transition while they follow the same scaling law i.e., the compounds have the same critical exponents. In contrast, for materials with a first-order phase transition- $\mathrm{DyCo}_{2}, \mathrm{HoCo}_{2}$, and $\mathrm{La}_{2 / 3} \mathrm{Ca}_{1 / 3} \mathrm{MnO}_{3}$ - the scaled
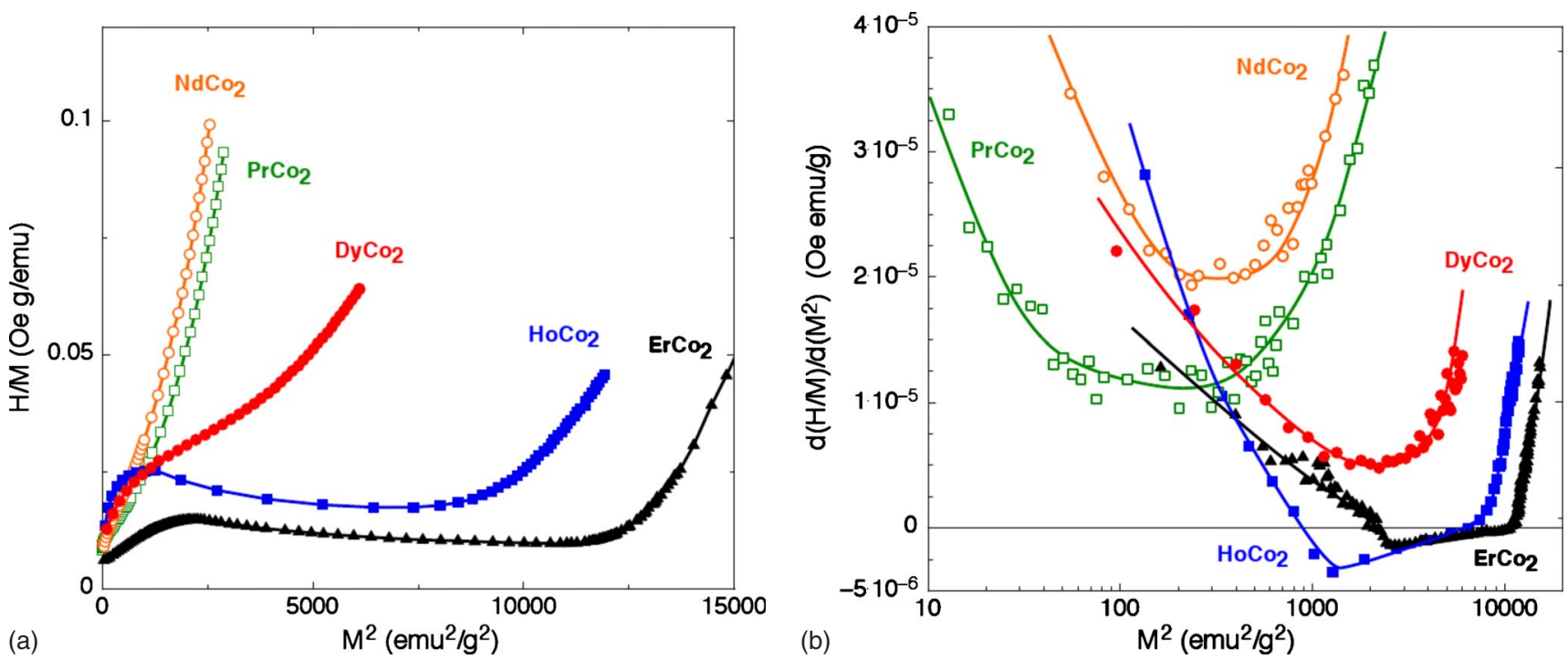

FIG. 5. (Color online) $H / M$ as function of $M^{2}$ (left) and derivative of $H / M$ vs $M^{2}$ curves (right), for $\mathrm{NdCo}_{2}$ (open circles), $\mathrm{PrCo}_{2}($ open squares), $\mathrm{DyCo}_{2}$ (full circles), $\mathrm{HoCo}_{2}$ (full squares), and $\mathrm{ErCo}_{2}$ (full triangles). 
$\Delta S_{M}$ curves do not follow a universal behavior. The difference between the scaled entropy change curves for compounds with first- and second-order phase transitions is clear from mere inspection regardless the family of compounds. Nevertheless, we have quantified the dispersion for the data in the ordered region. For compounds with second-order phase transition the dispersion is not over $27 \%$ while for compounds with first-order phase transition the dispersion remains well above $100 \%$ in all the cases. This result suggests the scaling of the entropy change curves as an alternative method for distinguishing the character of a transition from purely magnetic measurements.

\section{ACKNOWLEDGMENTS}

The financial support of MAT08-01077, DECRYPT, and CAMRADS is acknowledged. The authors are thankful to J. Bartolomé for fruitful discussions. C. M. Bonilla acknowledge a Banco Santander grant and a Spanish MICINN grant. V. Franco acknowledges the Spanish MICINN and EU FEDER (Project No. MAT 2007-65227), and the PAI of Regional Government of Andalucía (Project No. P06-FQM01823). *mbonilla@unizar.es

${ }^{1}$ V. Pecharsky and K. Gschneidner, J. Appl. Phys. 90, 4614 (2001).

${ }^{2}$ N. H. Duc and D. T. K. Anh, J. Magn. Magn. Mater. 242-245, 873 (2002)

${ }^{3}$ M. D. Kuz'min, Appl. Phys. Lett. 90, 251916 (2007).

${ }^{4}$ V. Franco, J. Blázquez, and A. Conde, Appl. Phys. Lett. 89, 222512 (2006).

${ }^{5}$ Q. Y. Dong, H. W. Zhang, J. R. Sun, B. G. Shen, and V. Franco, J. Appl. Phys. 103, 116101 (2006).

${ }^{6}$ V. Franco, C. Conde, J. Blázquez, and A. Conde, J. Appl. Phys. 101, 093903 (2007).

${ }^{7}$ V. Franco, C. Conde, J. S. Blázquez, M. Millán, and A. Conde, J. Appl. Phys. 102, 013908 (2007).

${ }^{8}$ V. Franco, C. F. Conde, A. Conde, and L. F. Kiss, Appl. Phys. Lett. 90, 052509 (2007).

${ }^{9}$ V. Franco, A. Conde, and L. Kiss, J. Appl. Phys. 104, 033903 (2008).

${ }^{10}$ V. Franco, J. Blázquez, and A. Conde, J. Appl. Phys. 103, $07 \mathrm{~B} 316$ (2008).

${ }^{11}$ V. Franco, A. Conde, V. Pecharsky, and K. A. Gschneidner, EPL 79, 47009 (2007).

${ }^{12}$ V. Franco, R. Caballero-Flores, A. Conde, Q. Dong, and H. Zhang, J. Magn. Magn. Mater. 321, 1115 (2009).

${ }^{13}$ N. H. Duc, P. E. Brommer, and K. H. J. Buschow, Handbook on Magnetic Materials (Elsevier, Amsterdam, 1999), Vol. 12, p. 259.

${ }^{14}$ E. Gratz and A. Markosyan, J. Phys.: Condens. Matter 13, R385 (2001).

${ }^{15}$ N. Duc, D. K. Anh, and P. Brommer, Physica B 319, 1 (2002).

${ }^{16}$ A. Giguere, M. Foldeaki, W. Schnelle, and E. Gmelin, J. Phys.: Condens. Matter 11, 6969 (1999).

${ }^{17}$ N. A. de Oliveira, P. J. von Ranke, M. V. Tovar Costa, and A. Troper, Phys. Rev. B 66, 094402 (2002).

${ }^{18}$ N. K. Singh, K. G. Suresh, A. K. Nigam, S. K. Malik, A. A. Coelho, and S. Gama, J. Magn. Magn. Mater. 317, 68 (2007).

${ }^{19}$ W. Dunhui, T. Shaolong, H. Songling, S. Zhenghua, H. Zhida, and D. Youwei, J. Alloys Compd. 360, 11 (2003).

${ }^{20}$ J. Herrero-Albillos, F. Bartolome, L. M. Garcia, F. Casanova, A. Labarta, and X. Batlle, Phys. Rev. B 73, 134410 (2006).
${ }^{21}$ J. Herrero-Albillos, F. Bartolome, L. M. Garcia, F. Casanova, A. Labarta, and X. Batlle, Phys. Rev. B 75, 187402 (2007).

${ }^{22}$ M. Parra-Borderias, F. Bartolome, J. Herrero-Albillos, and L. M. Garcia, J. Alloys Compd. 481, 48 (2009).

${ }^{23}$ J. Mira and J. Rivas, Mod. Phys. Lett. B 18, 725 (2004).

${ }^{24}$ J. Mira, J. Rivas, F. Rivadulla, C. Vázquez-Vázquez, and M. A. López-Quintela, Phys. Rev. B 60, 2998 (1999).

${ }^{25}$ M. H. Phan and S. C. Yu, Phys. Status Solidi A 204, 4091 (2007).

${ }^{26}$ C. A. Ramos, H. R. Salva, R. D. Sanchez, M. Tovar, F. Rivadulla, J. Mira, J. Rivas, A. M. Lopez-Quintela, L. Hueso, M. Saint-Paul, P. Lejay, and Y. Tokura, J. Magn. Magn. Mater. 226230, 582 (2001).

${ }^{27}$ M. Otero-Leal, I. Pardiñas, F. Rivadulla, M. A. López-Quintala, and J. Rivas, Bol. Soc. Esp. Ceram. Vidrio 45, 175 (2006).

${ }^{28}$ E. Burzo, A. Chelkowski, and H. R. Kirchmayr, LandoltBornstein, New Series Group III Vol. 19, Pt. d2 (SpirngerVerlag, Berlin, 1990).

${ }^{29}$ V. Franco, A. Conde, M. D. Kuz'min, and J. M. RomeroEnrique, J. Appl. Phys. 105, 07A917 (2009).

${ }^{30}$ V. Franco, A. Conde, J. M. Romero-Enrique, and J. S. Blázquez, J. Phys.: Condens. Matter 20, 285207 (2008).

${ }^{31}$ H. E. Stanley, Rev. Mod. Phys. 71, S358 (1999).

${ }^{32}$ B. Widom, J. Chem. Phys. 43, 3898 (1965).

${ }^{33}$ R. Caballero-Flores, V. Franco, A. Conde, and L. F. Kiss, J. Appl. Phys. 105, 07A919 (2009).

${ }^{34}$ J. L. Alonso, L. A. Fernández, F. Guinea, V. Laliena, and V. Martín-Mayor, Nucl. Phys. B 596, 587 (2001).

${ }^{35}$ S. Taran and B. K. Chaudhuri, J. Appl. Phys. 98, 103903 (2005).

${ }^{36}$ J. Inoue and M. Shimizu, J. Phys. F: Met. Phys. 12, 1811 (1982).

${ }^{37}$ M. Y. Efremov, J. T. Warren, E. A. Olson, M. Zhang, A. T. Kwan, and L. H. Allen, Macromolecules 35, 1481 (2002).

${ }^{38}$ J. Kamarad, Z. Arnold, and M. R. Ibarra, J. Magn. Magn. Mater. 140, 837 (1995).

${ }^{39}$ N. Ishimatsu, H. Maruyama, N. Kawamura, Y. Ohishi, and O. Shimomura, Nucl. Instrum. Methods Phys. Res. B 238, 167 (2005).

${ }^{40}$ K. Gu, J. Li, W. Ao, Y. Jian, and J. Tang, J. Alloys Compd. 441, 39 (2007)

${ }^{41}$ S. K. Banerjee, Phys. Lett. 12, 16 (1964). 